\title{
Infrequent mutations of the PPP2R1A and PPP2R1B genes in patients with ovarian cancer
}

\author{
FENG WANG $^{1^{*}}$, YANG ZOU ${ }^{*}$, FA-YING LIU ${ }^{1 *}$, XIAO-HONG YU ${ }^{2}$, HUANG HUANG ${ }^{3,4}$, NAN ZHANG $^{2}$, \\ YING-YING QI ${ }^{2}$, RONG-FANG LIU ${ }^{1,4}$, XIAO-YAN LIU ${ }^{1,4}$, JIA CHEN ${ }^{1,4}$, \\ OU-PING HUANG ${ }^{5}$ and $\mathrm{MING} \mathrm{HE}^{6}$ \\ ${ }^{1}$ Key Laboratory of Women's Reproductive Health of Jiangxi Province and ${ }^{2}$ Department of Pathology, \\ Jiangxi Provincial Maternal and Child Health Hospital; ${ }^{3}$ Department of Cardiothoracic Surgery, \\ The First Affiliated Hospital of Nanchang University, Nanchang, Jiangxi 330006; ${ }^{4}$ Graduate School of \\ Nanchang University, Nanchang, Jiangxi 330031; ${ }^{5}$ Jiangxi Provincial Cancer Institute, Jiangxi Provincial Cancer Hospital, \\ Nanchang, Jiangxi 330029; ${ }^{6}$ Department of Pharmacology and Molecular Therapeutics, \\ Nanchang University School of Pharmaceutical Science, Nanchang, Jiangxi 330006, P.R. China
}

Received January 16, 2013; Accepted March 27, 2013

DOI: $10.3892 / \mathrm{mmr} .2013 .1416$

\begin{abstract}
Protein phosphatase 2, regulatory subunit A, $\alpha$ $(P P P 2 R 1 A)$ and $\beta(P P P 2 R 1 B)$ are paralogous subunits of the heterotrimeric protein phosphatase 2 (PP2A) holoenzyme that catalyzes the dephosphorylation of target substrate proteins. Subtype-specific PPP2R1A mutations have been frequently observed in ovarian and endometrial cancer. Mutations in the paralogous genes were frequently observed in human malignancies. Thus, the present study aimed to analyze the mutation frequencies of the paralogous $P P P 2 R 1 A$ and $P P P 2 R 1 B$ genes in patients with primary and secondary ovarian cancer. A total of 251 patients with primary $(n=234)$ and secondary $(n=17)$ ovarian cancer were analyzed for the presence of $P P P 2 R 1 A$ and $P P P 2 R 1 B$ mutations by direct sequencing. For $P P P 2 R 1 A$, a heterozygous, somatic mutation (c.771G $>$ T, p.W257C) was identified in 1 out of 37 patients $(2.7 \%)$ with primary ovarian endometrioid carcinoma. The mutant sample was that of a 46-year-old female, who was also diagnosed with ectopic endometriosis in the benign ovary. No PPP $2 R 1 A$ mutations were detected in the remaining 250 patients with ovarian cancer. For $P P P 2 R 1 B$, no mutations were detected in our samples. The results of this study suggested that $P P P 2 R 1 A$
\end{abstract}

Correspondence to: Professor Ou-Ping Huang, Jiangxi Provincial Cancer Institute, Jiangxi Provincial Cancer Hospital, 519 Beijing East Road, Nanchang, Jiangxi 330029, P.R. China

E-mail: huangouping@gmail.com

Professor Ming He, Department of Pharmacology and Molecular Therapeutics, Nanchang University School of Pharmaceutical Science, 603 Bayi Road, Nanchang, Jiangxi 330006, P.R. China

E-mail: jxhm56@hotmail.com

${ }^{*}$ Contributed equally

Key words: PPP2R1A, PPP2R1B, mutation, Chinese, ovarian cancer mutations are less common in Chinese patients with ovarian cancer when compared with European and American patients. Furthermore, our study also supported previous observations that $P P P 2 R 1 B$ mutations were absent in ovarian cancer, suggesting that $P P P 2 R 1 B$ mutations are not actively involved in the pathogenesis of ovarian cancer.

\section{Introduction}

Protein phosphatase 2 , regulatory subunit A, $\alpha(P P P 2 R 1 A)$ and $\beta(P P P 2 R 1 B)$ are paralogous subunits of the heterotrimeric protein phosphatase 2 (PP2A) holoenzyme, which catalyzes the dephosphorylation of target substrate proteins (www.ensembl.org) (1). PPP $2 R 1 A$ and PPP $2 R 1 B$ belong to the Huntington-elongation-A subunit-TOR (HEAT) repeat protein family, and are required as scaffolds for the formation of the heterotrimeric PP2A complex (2). PPP2R1A and $P P P 2 R 1 B$ each contain 15 tandemly repeated HEAT motifs. It has been previously demonstrated that the HEAT repeats 2-8 and 11-15 were involved in binding to the regulatory and catalytic subunits of PP2A, respectively (3). In addition, mutations located in the HEAT 2 and 11 motifs promoted tumorigenesis; the mutant $P P P 2 R I A$ proteins were defective in binding to the regulatory and catalytic subunits of PP2A, resulting in a decrease in PP2A activity and thus facilitating tumor progression (4).

A previous whole-exome sequencing attempt identified recurrent $P P P 2 R 1 A$ mutations in 3 out of 42 patients $(7.1 \%)$ with ovarian clear cell carcinoma (OCCC) (5). Subsequent studies confirmed this finding and also revealed frequent $P P P 2 R 1 A$ mutations in patients with ovarian endometrioid carcinoma (6-8). Additionally, a high frequency of $P P P 2 R 1 A$ mutations was also identified in several subtypes of endometrial carcinoma, with the highest frequency demonstrated in the serous subtype (6-9). Furthermore, previous studies revealed that $P P P 2 R 1 A$ mutations were present in patients with breast and lung malignancies, but absent in patients with 
melanoma and Wilms' tumor $(10,11)$, suggesting a general effect of PPP2R1A mutations on human cancer. Until presently, the majority of $P P P 2 R 1 A$ mutations identified in human cancer were restricted to codons 179-183 and 256-258, which correspond to the HEAT 5 and HEAT 7 motifs, respectively (http://www.uniprot.org/) (5-9). Therefore, the PPP2R1A codons $179-183$ and 256-258 were considered potential mutational hotspots in human malignancies, particularly in ovarian and endometrial carcinoma.

$P P P 2 R 1 B$, the paralogous gene of $P P P 2 R 1 A$, was demonstrated to be mutated in breast and colorectal cancer, but not in ovarian cancer and certain other types of cancer $(10,12-21)$. Although point mutations in the $P P P 2 R 1 B$ gene have not been detected in ovarian cancer, it should be noted that a relatively small sample size was analyzed in these studies $(16,17)$, and loss of heterozygosity ( $\mathrm{LOH}$ ) of $P P P 2 R 1 B$ has been frequently observed in different subtypes of ovarian cancer (16). Additionally, accumulating evidence suggested that mutations in the homologous residues of paralogous genes were frequently observed in human malignancies (22-24), which suggested that $P P P 2 R 1 B$ mutations may also present in patients with ovarian cancer. We hypothesized that $P P P 2 R 1 A$ and $P P P 2 R 1 B$ mutations are also involved in the pathogenesis of non-primary ovarian cancer. Therefore, to examine whether PPP2RIA and PPP2RIB mutations may be involved in the development of primary and secondary ovarian cancer, 251 Chinese patients with diverse subtypes of ovarian cancer were recruited to test this hypothesis.

\section{Materials and methods}

Patients and clinical data. A total of 251 formalin-fixed, paraffin-embedded (FFPE) ovarian cancerous and paired normal tissues were recruited from the Jiangxi Provincial Maternal and Child Health Hospital (Jiangxi, China). The sections were reviewed in a blinded manner by two pathologists, and only those with $>70 \%$ cancerous cells were included in this study. In total, there were 234 primary and 17 secondary ovarian cancer samples. The primary ovarian cancer subtypes included ovarian serous cancer $(n=76)$, OCCC $(n=43)$, ovarian endometrioid carcinoma $(n=37)$, mucinous ovarian carcinoma $(n=15)$, ovarian germ cell tumor $(n=33)$, ovarian sex cord-stromal tumor $(n=18)$, other rare subtypes $(n=12)$ and secondary ovarian cancer $(n=17)$ (Table I); while all secondary types of cancer were Krukenberg tumors. This study conformed to the principles of the Declaration of Helsinki, and written informed consent was obtained from each subject prior to the study. The institutional review board of the Jiangxi Provincial Maternal and Child Health Hospital approved this study.

PPP2RIA and PPP2R1B mutational analyses. Genomic DNA was isolated from archival FFPE tissues using FFPE DNA kits (Omega Bio-Tek Inc., Doraville, GA, USA). For PPP2R1A, two short PCR fragments spanning codons 179-183 and 256-257 were amplified with the following primer pairs, respectively: Forward 1: 5'-GTACTTCCGGAACCTGTGCT-3' and reverse 1: 5'-AGCAAAACTCACCTGCTCGT-3'; forward 2: 5'-CTCTCCTCTCCCTAGGACTCG-3' and reverse 2: 5'-TGTGAACTTGTCAGCCACCA-3'. For $P P P 2 R 1 B$, the following primer pairs were used to amplify the PCR fragments spanning codons 191-195 and 268-269, corresponding to the homologous residues of codons 179-183 and 256-257 in PPP2R1A (Fig. 1): Forward 1: 5'-ATTCCGTTCCTTGTGCTCAG-3' and reverse 1: 5'-GGAGCATATCTGTGTCCCTTAAA-3'; forward 2: 5'-TAGGATTCAGTGCGCCTCCT-3' and reverse 2: 5'-TGAAAATCTGTCAGCCACCA-3'. The PCR products were sequenced using an ABI Prism 3730 DNA sequencer (Applied Biosystems, Foster City, CA, USA). The identified mutation was confirmed as either somatic or germline by sequencing the paired normal tissue.

Evolutionary conservation and protein sequence homology analyses. Evolutionary conservation analysis of the PPP2R1A mutation was performed using data obtained from GenBank for 14 different species, including Homo sapiens (PPP2R1A, GenBank accession no. NM_014225), Pan troglodytes (XM_001174546), Pongo abelii (NM_001132813), Macaca mulatta (NM_001257922.1), Nomascus leucogenys (XM_003269709), Callithrix jacchus (XM_002762433), Rattusnorvegicus(NM_057140),Musmusculus(NM_016891), Cricetulus griseus (XM_003509396), Canis lupus familiaris (XM_845900), Bos taurus (NM_001037477), Sus scrofa (NM_214024), Xenopus laevis (NM_001086666) and Danio rerio (NM_213376).

Protein sequence homology analysis of human PPP2R1A (GenBank accession no. NP_055040.2) and PPP2R1B (NP_859050.1) was performed to determine the relationship between the two paralogous genes, using DNASTAR software (DNASTAR, Inc., Madison, WI, USA).

\section{Results}

Patient characteristics. A total of 251 patients with primary and secondary ovarian cancer were recruited from the Department of Pathology, Jiangxi Provincial Maternal and Child Health Hospital. The median age of the patients was 47 years (range, 5-75), and 106 out of the 251 patients were affected in both ovaries, while the remaining 145 patients were affected in either the left or the right ovary (Table IA).

PPP2R1A mutations in ovarian cancer. We screened the 234 primary and 17 secondary ovarian cancer samples for the presence of PPP2R1A hotspot mutations. The mutation type and frequency distribution are shown in Table IB. A somatic, heterozygous PPP2R1A mutation (c.771G>T, p.W257C) was identified in 1 out of 37 primary ovarian cancer patients $(2.7 \%)$ with ovarian endometrioid carcinoma. The mutant sample was that of a 46-year-old female, who was also diagnosed with ectopic endometriosis in the benign ovary (Fig. 2). In addition, no PPP2R1A mutations were detected in the remaining 250 patients with ovarian cancer (Table IB).

The evolutionary conservation analysis suggested that the PPP2R1A p.W257C mutation was highly conserved among the 14 species ranging from Homo sapiens to Danio rerio (Fig. 3).

$P P P 2 R 1 B$ mutations in ovarian cancer. We sequenced the genomic region of $P P P 2 R 1 B$ spanning p.P191-R195 and p.S268-W269, the homologous residues corresponding to the potential mutational hotspots in the PPP2RIA gene. However, 
Table I. Patient characteristics and PPP2R1A mutation distribution in Chinese patients with primary and secondary ovarian cancer.

A. Patient characteristics.

\begin{tabular}{lr}
\hline Characteristics & Value \\
\hline Age at diagnosis (years) & 47 \\
Median & 5 \\
Minimum & 75 \\
Maximum & \\
Affected ovary (no. of patients) & 106 \\
Both & 63 \\
Left & 82 \\
Right
\end{tabular}

B. PPP2R1A mutation distribution.

\begin{tabular}{|c|c|c|c|}
\hline Cancer type & Frequency no. $(\%)$ & Nucleotide change & Amino acid change \\
\hline \multicolumn{4}{|l|}{ Primary } \\
\hline \multicolumn{4}{|l|}{ Epithelial } \\
\hline Serous & $0 / 76(0.0)$ & - & - \\
\hline Clear cell & $0 / 43(0.0)$ & - & - \\
\hline Endometrioid & $1 / 37(2.7)$ & c. $771 \mathrm{G}>\mathrm{T}$ & p.W257C \\
\hline Mucinous & $0 / 15(0.0)$ & - & - \\
\hline Undifferentiated & $0 / 3(0.0)$ & - & - \\
\hline Unclassified & $0 / 4(0.0)$ & - & - \\
\hline Transitional cell & $0 / 3(0.0)$ & - & - \\
\hline Mixed & $0 / 2(0.0)$ & - & - \\
\hline \multicolumn{4}{|l|}{ Non-epithelial } \\
\hline \multicolumn{4}{|l|}{ Germ cell tumor } \\
\hline Yolk sac & $0 / 11(0.0)$ & - & - \\
\hline Dysgerminoma & $0 / 7(0.0)$ & - & - \\
\hline Teratoma & $0 / 9(0.0)$ & - & - \\
\hline Mixed & $0 / 6(0.0)$ & - & - \\
\hline \multicolumn{4}{|c|}{ Sex cord-stromal tumor } \\
\hline Granulosa cell & $0 / 16(0.0)$ & - & - \\
\hline Sertoli-Leydig & $0 / 2(0.0)$ & - & - \\
\hline \multicolumn{4}{|l|}{ Secondary } \\
\hline Krukenberg tumors & $0 / 17(0.0)$ & - & - \\
\hline Total & $251(100)$ & c. $771 \mathrm{G}>\mathrm{T}$ & p.W257C \\
\hline
\end{tabular}

PPP2R1A, protein phosphatase 2, regulatory subunit A, $\alpha$.

no mutations were detected among the 234 primary and 17 secondary patients with ovarian cancer.

\section{Discussion}

Recurrent PPP2R1A mutations have been identified in OCCC patients in a whole-exome sequencing study (5). This observation was confirmed by four studies that followed, and frequent PPP2R1A mutations were also identified in several other subtypes of ovarian and endometrial carcinoma (6-9). In addition, previous studies detected $P P P 2 R 1 A$ and $P P P 2 R 1 B$ mutations in human malignancies with variable frequencies $(10-21,25)$.

In the present study, 234 primary and 17 secondary Chinese patients with ovarian cancer were analyzed for the presence of potential $P P P 2 R 1 A$ and $P P P 2 R 1 B$ hotspot mutations. A previously reported PPP2R1A p.W257C (c.771G $>$ T) somatic mutation was detected in the sample from one patient with ovarian endometrioid carcinoma out of our 251 samples. This particular patient was also diagnosed with ectopic endo- 
179-183

PPP2R1A (NP_055040.2)

PPP2R1B (NP_859050.1)

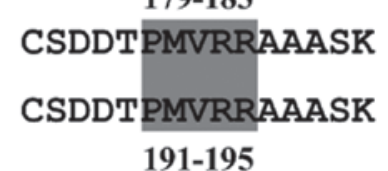

256-257

PPP2R1A (NP_055040.2)

PPP2R1B (NP_859050.1)

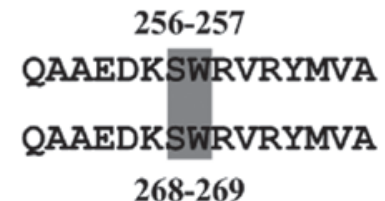

Figure 1. Protein sequence homology analysis of protein phosphatase 2, regulatory subunit A, $\alpha$ (PPP2R1A) and $\beta$ (PPP2R1B). Underlined, affected codons.

Codon

255

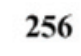

257

258

259

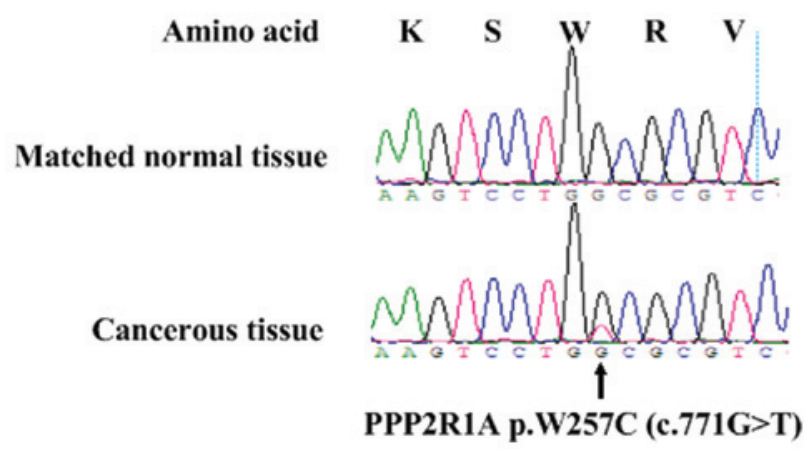

Figure 2. Sequencing electropherograms of the protein phosphatase 2, regulatory subunit $\mathrm{A}, \alpha(P P P 2 R 1 A)$ p.W257C mutation. The arrow refers to the location of the mutation.
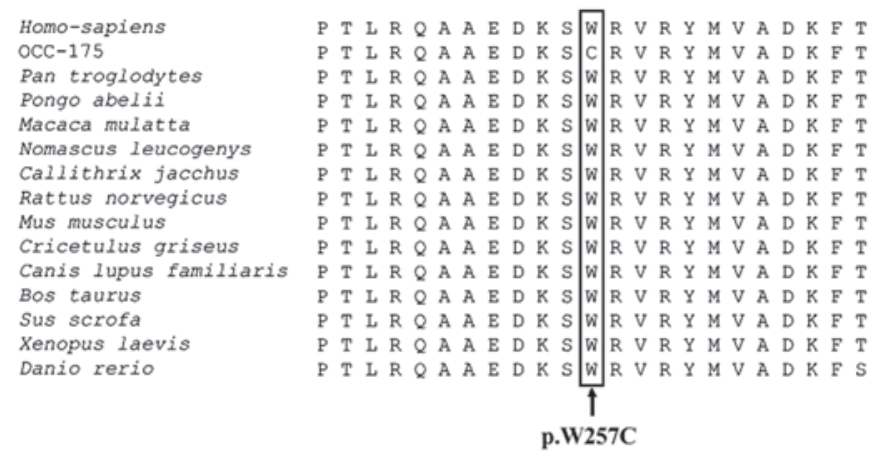

Figure 3. Evolutionary analysis of the protein phosphatase 2, regulatory subunit A, $\alpha(P P P 2 R 1 A)$ p.W257C mutation. Patient 'OCC-175' harbored the PPP2R1A p.W257C mutation, as indicated by the arrow.

metriosis in the benign ovary. We failed to detect the presence of any mutations in the remaining individuals. In addition, no $P P P 2 R 1 B$ mutations were detected in our samples.

The combined results of previous studies demonstrated that PPP2R1A mutations frequently occurred in breast, lung and endometrial carcinoma, as well as in OCCC and ovarian endometrioid carcinoma (5-11). Furthermore, the mutation frequencies of PPP2RIA in OCCC and ovarian endometrioid carcinoma patients were $4.1-9.1 \%$ (mean, $7.4 \%$ ) and $10.0-12.2 \%$ (mean, 11.1\%), respectively (5-8). However, the mutation frequencies of $P P P 2 R 1 A$ in our OCCC and ovarian endometrioid carcinoma samples were $0 \%(0 / 43)$ and $2.7 \%(1 / 37)$, respectively, which were lower than those observed in the European and American counterparts (5-8). One reason for these discrepancies may be population differences, suggesting that PPP2RIA mutations may increase the propensity for ovarian cancer when present in European and American individuals compared with Chinese individuals. An alternative explanation for these differences may be the relatively small sample size analyzed in our study, therefore, larger sample sizes would be required to test this theory. Moreover, we did not detect any PPP2R1A mutations in OSC or ovarian mucinous carcinoma, which was consistent with previous observations $(6,8)$.

A previous study demonstrated that PPP2R1A p.E64D, p.E64G and p.R418W mutations located in the HEAT 2 and 11 motifs, respectively, contributed to carcinogenesis by impairing the activity of PP2A (http://www.uniprot.org/) (4). Mutations of the PPP2R1A codon 257 have been frequently observed in ovarian cancer (6-8), and are highly conserved across multiple species (Fig. 3). Additionally, the PPP2R1A p.W257C mutation was located in the HEAT 7 repeat motif (http://www.uniprot.org/), which has been proposed to be the domain interacting with the regulatory subunits of PP2A, and thus crucial for PP2A activity (3). Overall, we speculated that the PPP2R1A p.W257C mutation may contribute to the pathogenesis of Chinese patients with ovarian endometrioid carcinoma. Nevertheless, the pathogenicity of the PPP2R1A p.W257C mutation remains unclear, therefore, functional assays are required to decipher the potential role of this mutation in the development of ovarian cancer.

We also screened for potential mutations of p.P191-R195 and p.S268-W269, the homologous residues corresponding to potential mutational hotspots in the PPP2R1A gene. However, no mutations were evident in our samples. This was consistent with previous observations that $P P P 2 R 1 B$ mutations were absent in patients with ovarian cancer $(16,17)$. Combined with the results of previous studies $(16,17)$, the absence of $P P P 2 R 1 B$ mutations in the ovarian cancer samples analyzed in our study suggested that $P P P 2 R 1 B$ mutations may not be actively involved in the development of primary and secondary ovarian cancer. However, this conclusion should be treated with caution, as the most frequent genetic aberrations of $P P P 2 R 1 B$ observed in human cancers were heterozygous or homozygous $\mathrm{LOH}(10,19)$. Furthermore, we did not exclude the possibility that there may be mutations in other regions of this gene contributing to the development of ovarian cancer.

In conclusion, we analyzed 251 Chinese patients with primary and secondary ovarian cancer for the presence of $P P P 2 R 1 A$ and $P P P 2 R 1 B$ mutations. The mutation frequencies of PPP2R1A were 0.0 and $2.7 \%$ in OCCC and ovarian endometrioid carcinoma patients, respectively, which were lower than those of their corresponding European and American counterparts. In addition, we did not detect any $P P P 2 R 1 A$ mutations in OSC and ovarian mucinous carcinoma, which was consistent with previous observations. Moreover, no $P P P 2 R 1 B$ mutations were identified in our samples, and this observation was consistent with previous studies on ovarian 
cancer. Our results suggested that mutations of $P P P 2 R 1 A$, but not $P P P 2 R 1 B$, may be involved in the pathogenesis of Chinese patients with ovarian cancer.

\section{Acknowledgements}

The authors would like to thank the sample donors who participated in this study. This study was supported by the National Natural Science Foundation of China (grant nos. 81060052 and 81260384) and the Natural Science Foundation of Jiangxi Province (grant no. 20114BAB215033).

\section{References}

1. Slupe AM, Merrill RA and Strack S: Determinants for substrate specificity of protein phosphatase 2A. Enzyme Res 2011: 398751, 2011.

2. Ruediger R, Roeckel D, Fait J, Bergqvist A, Magnusson G and Walter G: Identification of binding sites on the regulatory $A$ subunit of protein phosphatase $2 \mathrm{~A}$ for the catalytic $\mathrm{C}$ subunit and for tumor antigens of simian virus 40 and polyomavirus. Mol Cell Biol 12: 4872-4882, 1992.

3. Xu Y, Xing Y, Chen Y, Chao Y, Lin Z, Fan E, Yu JW, Strack S, Jeffrey PD and Shi Y: Structure of the protein phosphatase 2A holoenzyme. Cell 127: 1239-1251, 2006.

4. Chen W, Arroyo JD, Timmons JC, Possemato R and Hahn WC: Cancer-associated PP2A Aalpha subunits induce functional haploinsufficiency and tumorigenicity. Cancer Res 65: 8183-8192, 2005.

5. Jones S, Wang TL, Shih IM, Mao TL, Nakayama K, Roden R, Glas R, Slamon D, Diaz LA Jr, Vogelstein B, et al: Frequent mutations of chromatin remodeling gene ARID1A in ovarian clear cell carcinoma. Science 330: 228-231, 2010.

6. McConechy MK, Anglesio MS, Kalloger SE, Yang W, Senz J, Chow C, Heravi-Moussavi A, Morin GB, Mes-Masson AM; Australian Ovarian Cancer Study Group, et al: Subtype-specific mutation of PPP2R1A in endometrial and ovarian carcinomas. J Pathol 223: 567-573, 2011.

7. Nagendra DC, Burke J III, Maxwell GL and Risinger JI: PPP2R1A mutations are common in the serous type of endometrial cancer. Mol Carcinog 51: 826-831, 2012.

8. Shih IM, Panuganti PK, Kuo KT, Mao TL, Kuhn E, Jones S, Velculescu VE, Kurman RJ and Wang TL: Somatic mutations of PPP2R1A in ovarian and uterine carcinomas. Am J Pathol 178: 1442-1447, 2011

9. McConechy MK, Ding J, Cheang MC, Wiegand KC, Senz J, Tone AA, Yang W, Prentice LM, Tse K, Zeng T, et al: Use of mutation profiles to refine the classification of endometrial carcinomas. J Pathol 228: 20-30, 2012.

10. Calin GA, di Iasio MG, Caprini E, Vorechovsky I, Natali PG, Sozzi G, Croce CM, Barbanti-Brodano G, Russo G and Negrini M: Low frequency of alterations of the alpha (PPP2R1A) and beta (PPP2R1B) isoforms of the subunit A of the serine-threonine phosphatase 2A in human neoplasms. Oncogene 19: 1191-1195, 2000 .
11. Ruteshouser EC, Ashworth LK and Huff V: Absence of PPP2R1A mutations in Wilms tumor. Oncogene 20: 2050-2054, 2001.

12. Wang SS, Esplin ED, Li JL, Huang L, Gazdar A, Minna J and Evans GA: Alterations of the PPP2R1B gene in human lung and colon cancer. Science 282: 284-287, 1998.

13. Takagi Y, Futamura M, Yamaguchi K, Aoki S, Takahashi T and Saji S: Alterations of the PPP2R1B gene located at 11q23 in human colorectal cancers. Gut 47: 268-271, 2000.

14. Tamaki M, Goi T, Hirono Y, Katayama K and Yamaguchi A PPP2R1B gene alterations inhibit interaction of PP2A-Abeta and PP2A-C proteins in colorectal cancers. Oncol Rep 11: 655-659, 2004.

15. Suraweera N, Robinson J, Volikos E, Guenther T, Talbot I, Tomlinson I and Silver A: Mutations within Wnt pathway genes in sporadic colorectal cancers and cell lines. Int J Cancer 119: 1837-1842, 2006.

16. Wu R, Connolly DC, Ren X, Fearon ER and Cho KR: Somatic mutations of the PPP2R1B candidate tumor suppressor gene at chromosome 11q23 are infrequent in ovarian carcinomas. Neoplasia 1: 311-314, 1999.

17. Campbell IG and Manolitsas T: Absence of PPP2R1B gene alterations in primary ovarian cancers. Oncogene 18: 6367-6369, 1999.

18. Marsh A, Healey S, Lewis A, Spurdle AB, Kedda MA, Khanna KK; kConFab, Mann GJ, Pupo GM, Lakhani SR and Chenevix-Trench G: Mutation analysis of five candidate genes in familial breast cancer. Breast Cancer Res Treat 105: 377-389, 2007.

19. Chou HC, Chen CH, Lee HS, Lee CZ, Huang GT, Yang PM, Lee PH and Sheu JC: Alterations of tumour suppressor gene PPP2R1B in hepatocellular carcinoma. Cancer Lett 253: 138-143, 2007.

20. Hemmer S, Wasenius VM, Haglund C, Zhu Y, Knuutila S, Franssila $\mathrm{K}$ and Joensuu H: Alterations in the suppressor gene PPP2R1B in parathyroid hyperplasias and adenomas. Cancer Genet Cytogenet 134: 13-17, 2002.

21. Yeh LS, Hsieh YY, Chang JG, Chang WW, Chang CC and Tsai FJ: Mutation analysis of the tumor suppressor gene PPP2R1B in human cervical cancer. Int J Gynecol Cancer 17: 868-871, 2007.

22. Su F, Viros A, Milagre C, Trunzer K, Bollag G, Spleiss O, Reis-Filho JS, Kong X, Koya RC, Flaherty KT, et al: RAS mutations in cutaneous squamous-cell carcinomas in patients treated with BRAF inhibitors. N Engl J Med 366: 207-215, 2012.

23. Van Raamsdonk CD, Griewank KG, Crosby MB, Garrido MC, Vemula S, Wiesner T, Obenauf AC, Wackernagel W, Green G, Bouvier N, et al: Mutations in GNA11 in uveal melanoma. N Engl J Med 363: 2191-2199, 2010.

24. Zou Y, Zeng Y, Zhang DF, Zou SH, Cheng YF and Yao YG: IDH1 and IDH2 mutations are frequent in Chinese patients with acute myeloid leukemia but rare in other types of hematological disorders. Biochem Biophys Res Commun 402: 378-383, 2010

25. Zhu Y, Loukola A, Monni O, Kuokkanen K, Franssila K, Elonen E, Vilpo J, Joensuu H, Kere J, Aaltonen L and Knuutila S: PPP2R1B gene in chronic lymphocytic leukemias and mantle cell lymphomas. Leuk Lymphoma 41: 177-183, 2001. 